OPEN ACCESS

Edited by:

Philip Norris,

Blood Systems, United States

Reviewed by:

Namal P. M. Liyanage,

Ohio State University, United States

Lucia Lopalco,

San Raffaele Hospital (IRCCS), Italy

${ }^{*}$ Correspondence: Hong Shang hongshang100@hotmail.com: Zi-Ning Zhang

zi_ning101@hotmail.com

Specialty section: This article was submitted to HIV and AIDS,

a section of the journal

Frontiers in Immunology

Received: 25 May 2017 Accepted: 25 August 2017 Published: 11 September 2017

Citation:

Wu X, Zhang L-L, Yin L-B, Fu Y-J, Jiang $Y-J$, Ding $H-B$, Chu Z-X, Shang $H$ and Zhang Z-N (2017) Deregulated MicroRNA-21 Expression in Monocytes from HIV-Infected Patients Contributes

to Elevated IP-10 Secretion in HIV Infection.

Front. Immunol. 8:1122. doi: 10.3389/fimmu.2017.01122

\section{Deregulated MicroRNA-21 Expression in Monocytes from HIV-Infected Patients Contributes to Elevated IP-10 Secretion in HIV Infection}

\author{
Xian Wu $\mathbf{W}^{1,2}$, Le-Le Zhang ${ }^{1,2}$, Lin-Bo Yin 1,2, Ya-Jing Fu',2, Yong-Jun Jiang ${ }^{1,2}$, Hai-Bo Ding ${ }^{1,2}$, \\ Zhen-Xing Chu ${ }^{1,2}$, Hong Shang ${ }^{1,2 *}$ and Zi-Ning Zhang ${ }^{1,2 *}$ \\ ${ }^{1}$ Key Laboratory of AIDS Immunology of National Health and Family Planning Commission, Department of Laboratory \\ Medicine, The First Affiliated Hospital, China Medical University, Shenyang, China, ${ }^{2}$ Collaborative Innovation Center for \\ Diagnosis and Treatment of Infectious Diseases, Hangzhou, China
}

Persistent activation and inflammation impair immune response and trigger disease progression in HIV infection. Emerging evidence supports the supposition that excessive production of interferon-inducible protein 10 (IP-10), a critical inflammatory cytokine, leads to immune dysfunction and disease progression in HIV infection. In this study, we sought to elucidate the cause of the upregulated production of IP-10 in HIV infection and explore the underlying mechanisms. Bolstering miR-21 levels using mimics resulted in the obvious suppression of lipopolysaccharide (LPS)-induced IP-10 in monocyte leukemia cells THP-1 and vice versa. The analysis of the primary monocytes of HIV patients revealed significantly less miR-21 than in healthy controls; this was opposite to the tendency of IP-10 levels in plasma. The secretion of IP-10 due to LPS stimulation was not affected by miR-21 modulation in the differentiated THP-1 macrophages (THP-1-MA). We found a novel switch, IFN-stimulated gene 15 (ISG15), which triggers the expression of IP-10 and is significantly upregulated during the differentiation of THP-1 into THP1-MA. The inhibition of ISG15 can restore the regulation of IP-10 by miR-21. In summary, $\mathrm{IP}-10$ expression in monocytes is regulated by miR-21, whereas in macrophages, this fine-tuning is attenuated by the enhanced expression of ISG15. This study paves the way to a comprehensive understanding of the molecular regulatory mechanism of IP-10, a key point in immune intervention strategy.

Keywords: interferon-inducible protein 10, microRNA-21, IFN-stimulated gene 15, inflammatory response, HIV infection

\section{INTRODUCTION}

The deterioration of the gut epithelium and enlarged microbial translocation triggered by the rapid depletion of gastrointestinal $\mathrm{CD} 4^{+} \mathrm{T}$ cells leads to a persistent, systemic activation, and inflammation of HIV infections $(1,2)$. Antiretroviral therapy (ART) extends the lifespan and the quality of life of HIV-infected patients while completely eliminating chronic immune activation and inflammation does not occur $(3,4)$. Persistent inflammation contributes to immune senescence and exhaustion, factors linked to most non-AIDS-related complications of HIV, such as cardiovascular, 
neurocognitive, and metabolic syndromes (2-5). A comprehensive understanding of the molecular and cellular basis of the inflammatory status allows these remaining challenges of HIV treatment to be addressed.

Excessive chronic production of inflammatory cytokines, including TNF- $\alpha$, IFN- $\gamma$, interleukin (IL)-18, and interferoninducible protein 10 (IP-10), has been recorded in HIV-infected subjects (6-8); among these, IP-10 is functionally categorized as an inflammatory chemokine which attributed to inflammatory disorders, immune dysfunction, and tumor development (9). In HIV infection, elevated blood levels of IP-10 are associated with rapid disease progression and persistent immune activation $(6,10-12)$. Previous studies found that IP-10 decreased the function of T cells (13) and NK cells (14) and stimulated HIV replication (15). This indicates that the regulation of IP-10 expression in HIV infection influences the delay of inflammation, slowing down disease progression. Studies have shown that HIV-1 infection induces the production of IP-10 through the TLR7/9 dependent (16) or MDA5/Rig-I pathways (17). However, the factors triggering the significant upregulation of IP-10, especially at a post-transcriptional level in HIV infection have not been clearly elucidated.

The correlation between microRNAs (miRNAs), a species of small RNA molecules that are important in gene regulation, and HIV-1 infection emerged as a promising platform to understand the complex molecular mechanisms that occur in infection (18). More and more evidence supports the contention that the changes within the endogenous miRNA levels observed after HIV infection are essential to controlling HIV-1 replication and latency (19) and modulating the key targets associated with T cell exhaustion (20). Several previous studies have shown multiple processes of cross-regulation between cytokine and the miRNA pathways (21). In addition, miRNA influences immune function by inducing changes in cytokine, such as interleukin-2 (IL-2) and IL-10 in HIV infection $(22,23)$. The potential of miRNA to regulate IP-10 in HIV infection has not yet been reported.

In this study, we investigated miRNAs that might target IP-10 and suppress its level in monocytes. We found that miR-21 could target and suppress the secretion of IP-10 in monocytes and that HIV-infected patients exhibited a decreased level of miR-21 in monocytes. However, the modulation of the expression of miR-21 had no effect on IP-10 in macrophages, which is partly ascribed to the elevated expression levels of ISG15 in these cells. The data showed that miR-21 regulated levels of IP-10, which paves the way to a comprehensive understanding of the molecular regulatory mechanism of IP-10.

\section{MATERIALS AND METHODS}

\section{Patient Selection}

In total, 32 treatment-naive HIV-infected patients and 35 healthy controls (HCs) participated in this study. The demographic information and clinical characteristics of the subjects are listed in Table 1. Peripheral blood mononuclear cells (PBMCs) were freshly isolated by Ficoll centrifugation, and plasma samples were collected. Ethical approval was obtained from the First Hospital of China Medical University, and all participants were informed
TABLE 1 | Demographic and clinical characteristics of subjects.

\begin{tabular}{lcc}
\hline Characteristic & $\begin{array}{c}\text { HIV infected } \\
\text { patients }\end{array}$ & $\begin{array}{c}\text { Normal } \\
\text { controls }\end{array}$ \\
\hline Subject no. & 32 & 35 \\
Age (years, mean $\pm \mathrm{SD})$ & $34 \pm 6$ & $36 \pm 6$ \\
Male (No, \%) & $15(46.9 \%)$ & $15(42.9 \%)$ \\
Han Ethnic (No, \%) & $32(100 \%)$ & $35(100 \%)$ \\
CD4 (cells/ $\mu \mathrm{L}$, mean $\pm \mathrm{SD})$ & $331 \pm 235$ & $\mathrm{~N} / \mathrm{A}$ \\
VL [log copies/mL (mean $\pm \mathrm{SD})]$ & $4.15 \pm 1.09$ & $\mathrm{~N} / \mathrm{A}$ \\
Time since diagnosis (weeks, mean, range) & $65(1-336)$ & $\mathrm{N} / \mathrm{A}$ \\
\hline
\end{tabular}

of the collection of blood samples and provided written consent prior to enrolment in the study.

\section{Primary Cells Isolation and THP-1 Cell Culture}

For primary cell isolation, monocytes $\left(\mathrm{CD}^{-} \mathrm{CD} 14^{+}\right)$, NK cells $\left(\mathrm{CD}^{-}{ }^{-} \mathrm{CD} 56^{+} \mathrm{CD}^{-} 6^{+}\right), \mathrm{CD}^{+} \mathrm{T}$ cells $\left(\mathrm{CD} 3^{+} \mathrm{CD} 4^{+}\right), \mathrm{CD}^{+} \mathrm{T}$ cells $\left(\mathrm{CD}^{+}{ }^{+} \mathrm{CD} 8^{+}\right)$, and $\mathrm{B}$ cells $\left(\mathrm{CD} 3^{-} \mathrm{CD} 19^{+}\right)$were selected from PBMCs using a BD FACS Aria flow cytometer. THP-1 human acute monocyte leukemia cells were cultured in RPMI 1640 supplemented with 10\% FBS (HyClone). To promote the differentiation of THP-1 to THP-1 macrophages (THP-1-MA) cells, the THP-1 were treated with phorbol 12-myristate 13-acetate (PMA; $100 \mathrm{ng} / \mathrm{mL}$; Sigma) for $48 \mathrm{~h}$ (24).

\section{miRNA Mimics, Inhibitors, and siRNA Delivery}

The transfection of miRNA mimics, inhibitors, and controls (GenePharma) were performed with HiperFect Transfection Reagent (Qiagen). Briefly, $20 \mu \mathrm{M}$ mimics or inhibitors were transfected into THP-1, THP-1-MA cells for 48 h. Subsequently, the THP-1 cells were stimulated using lipopolysaccharide (LPS; $1 \mu \mathrm{g} / \mathrm{mL}$; Sigma), and incubated for a further $24 \mathrm{~h}$. The cells were collected for RNA extraction, and the supernatants were collected for IP-10 detection. Transfection of primary CD $14^{+}$monocytes with mimics was performed with RNAiMAX (Invitrogen) according to the manufacturer's protocol. The sequences of the mimics and inhibitors are listed in Table S1 in Supplementary Material. The forced reduction process of ISG15 was achieved by employing the $20 \mu \mathrm{M}$ ISG15 siRNA for $24 \mathrm{~h}$ (Invitrogen). This was followed by THP- 1 cell treatment with PMA for $48 \mathrm{~h}$, into which was transfected $20 \mathrm{nM}$ mimics by HiperFect Transfection Reagent (Qiagen). For the inhibition of ISG15 in THP-1 cells, IG15 siRNA was transfected to THP-1 cells for $24 \mathrm{~h}$. The siRNA control was represented by non-specific Stealth $\mathrm{RNAi}^{\circledR}$ Negative Control Duplexes.

\section{Reverse Transcription and Quantitative Real-time PCR}

MicroRNA was extracted from cells using the miRNeasy Micro kit (Qiagen). Total RNA was isolated using the RNeasy Micro kit (Qiagen), and the purified RNA was treated with DNase I reagent to eliminate genomic DNA contamination. The RNA was reversely transcribed using Primpscript ${ }^{\circledR} \mathrm{RT}$ reagent kit (TAKARA) according to the instructions provided by the 
manufacturer. The real-time PCRs for the detection of miRNA and mRNA were performed with SYBR ${ }^{\circledR}$ Premix Ex Taq ${ }^{\mathrm{TM}}$ II (TAKARA). All the primer sequences are listed in Table $\mathrm{S} 1$ in Supplementary Material. The levels of miRNA expression were normalized to small nucleolar RNA (snRU6), while mRNA expression was normalized to GAPDH. The relative expression levels of miRNA and mRNA were calculated based on the change in cycling threshold method as $2^{-\Delta \Delta C t}$.

\section{IP-10 Detection}

The measurements of supernatant IP-10 produced from cell culture and plasma from $\mathrm{HIV}$-infected patients and HCs were performed using an ELISA kit (R\&D Systems). Concentrations of IP-10 were calculated using the standard curve and multiplied by the dilution factor.

\section{Luciferase Activity Assay}

According to the target prediction, the IP-10 3'UTR contained two appropriate sequences with miR-21 binding sites (196-202 and 235-240 nt). The 3'UTR fragments (synthesized by GenePharma), containing the putative binding sites for miR-21, were cloned into the GP-miRGLO Vector. The luciferase reporter assay was performed in $293 \mathrm{~T}$ cells cultured in 48 -well plates. The $293 \mathrm{~T}$ cells were co-transfected with the IP-10 3'UTR reporter (wild type, mut-1, mut-2), empty vector plasmid (GP-miRGLO), and appropriate miRNA mimics and inhibitors $(20 \mu \mathrm{M})$, using the Lipofectamine 2000 reagent (Invitrogen). At $24 \mathrm{~h}$ after transfection, firefly and renilla luciferase activities were measured using the Dual-Luciferase Report Assay (Promega).

\section{Statistical Analysis}

GraphPad Prism was used to conduct statistical analyses, and the comparison between the miRNA and IP-10 levels was performed using the Student's $t$-test. The non-parametric Mann-Whitney test was used to determine the differences between HIV patients and HCs. The correlations between variables were evaluated using the Spearman's correlation coefficient, and all $P$ values under 0.05 were considered statistically significant.

\section{RESULTS}

\section{Elevated IP-10 Levels in HIV-Infected Patients Are Linked to Disease Progression}

First, we investigated levels of IP-10 secretion in the HIV-infected patients enrolled in our study. All of these patients had considerably greater IP-10 plasma concentrations $(458.0 \pm 329.5 \mathrm{pg} / \mathrm{mL})$ than HCs $(115.0 \pm 56.3 \mathrm{pg} / \mathrm{mL})(P<0.0001$; Figure $\mathrm{S} 1$ in Supplementary Material). Then we studied the association of levels of IP-10 with disease progression. We found that IP-10 was negatively correlated with $\mathrm{CD} 4^{+} \mathrm{T}$-cell counts $(r=-0.595$, $P=0.0003$; Figure 1A) and positively correlated with viral load $(r=0.706, P<0.0001$; Figure 1B). We then divided the patients
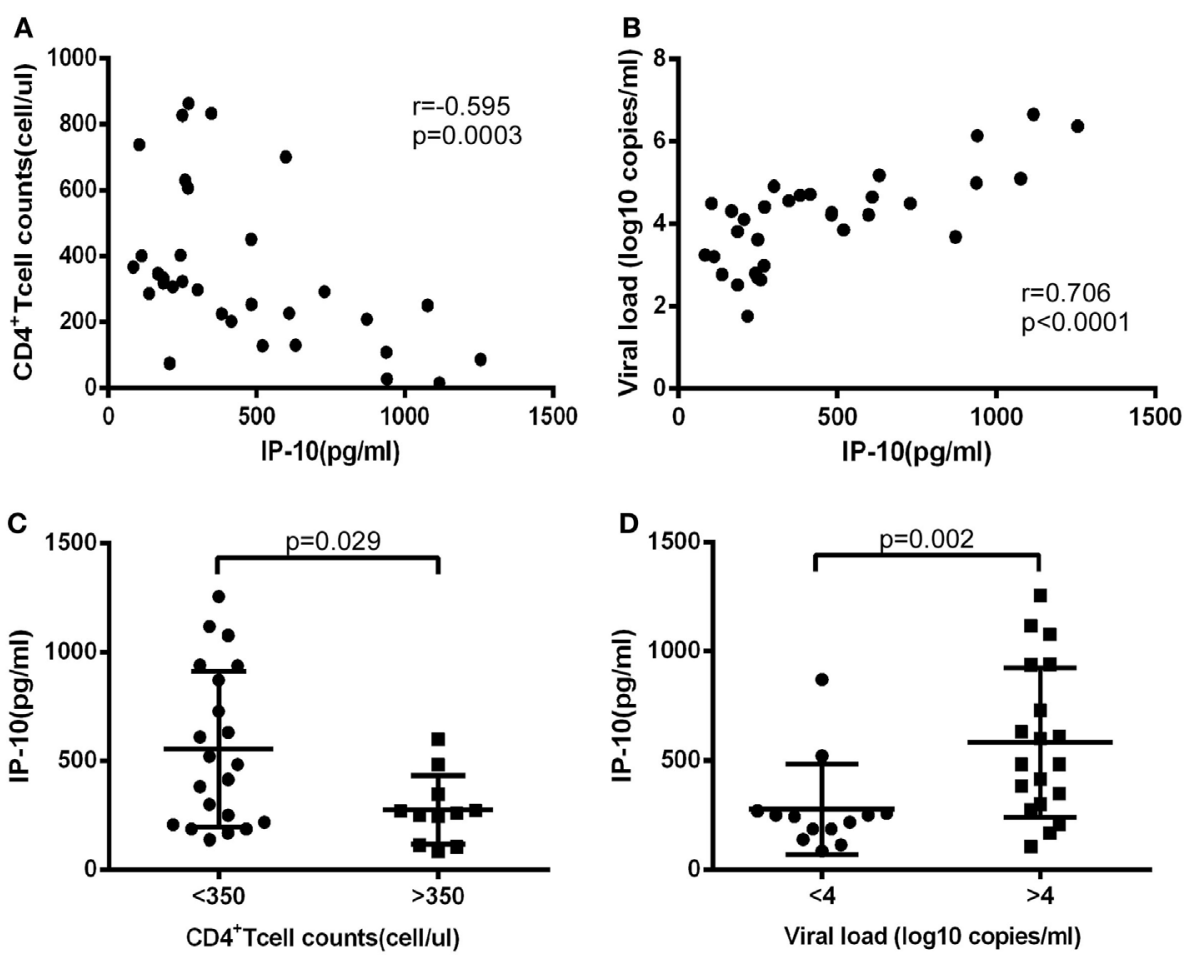

FIGURE 1 The increased interferon-inducible protein 10 (IP-10) level was association with progression of HIV infection. The relationship between plasma IP-10 and CD4+ T cell counts (A), viral load (B) in HIV-infected patients; (C) comparison of plasma IP-10 in HIV-infected patients in high $(>350$ cells/ $\mu \mathrm{L})$ and low $(<350$ cells/ $\mu \mathrm{L})$ $\mathrm{CD}^{+} \mathrm{T}$ cell count groups; (D) comparison of plasma IP-10 in HIV-infected patients in low (<4 log copies/mL) and high (>4 log copies/mL) viral load groups. 
into two groups according to $\mathrm{CD}^{+} \mathrm{T}$ cell counts and viral load. IP-10 levels were significantly higher in the group of patients with seriously reduced $\mathrm{CD} 4^{+} \mathrm{T}$ cell counts $(<350$ cells $/ \mu \mathrm{L} ; P=0.029$; Figure 1C) and the group with high viral loads ( $\operatorname{LogVL}>4$; $P=0.002$; Figure 1D). The correlation between IP-10 and disease progression confirmed that IP-10 is a good biomarker in HIV disease progression.

\section{Identification of Individual miRNAs for IP-10 Production Regulation}

Although the key role played by IP-10 in HIV infection has been recognized, the factors regulating its expression, particularly at post-transcriptional level, have not been investigated. Given that miRNAs act as repressors and have the propensity to fine-tune gene expression, we sought to explore whether miRNAs can regulate IP-10 expression. Three miRNA target prediction tools were employed to identify miRNA candidates: TargetScan, miRanda, and DIANA. These online tools showed that six miRNAs possessed a conserved 6-7 mer seed matching the IP-10 3'UTR. These were miR-15a, miR-16, miR-21, miR-135a, miR-200c, and miR-503. As illustrated in Figure 2A, IP-10 exhibited two binding sites for miR-21 and just one for the other miRNAs.

Early HIV-infected patients, whose durations of infection were $0-40$ weeks, display considerably distinct IP-10 mRNA levels among $\mathrm{T}$ cells, mDCs, monocytes, and B-cells, according to previously reported studies (16). Thus, we initially investigated the variation of IP-10 mRNA levels of expression among a series of cells, sorted B-cells, monocytes, $\mathrm{NK}, \mathrm{CD}^{+}$, and $\mathrm{CD}^{+} \mathrm{T}$ cells supplied by healthy donors, and found that monocytes had the highest levels of expression of IP-10 mRNA (Figure 2B), which is consistent with previous studies. Therefore, THP-1 cells, a human acute monocyte leukemia cell line, were selected to perform the in vitro experiments. The THP-1 cells were overexpressed for the six miRNA candidates and stimulated with LPS (25) to identify the miRNAs which could regulate IP-10 production. IP-10 secretion was largely reduced by the overexpression of miR-21 $(P=0.003)$ and miR-200c $(P<0.0001)$ compared to the control (Figure 2C). Because the overexpression multiples of miR-200c were too high (data not shown) to be achieved in vivo, only miR21 was identified for further studies.

\section{miR-21 Regulates IP-10 Secretion through THP-1 Cells}

Next, we transfected miR-21 mimics to the THP-1 cells (Figure 3A), finding that miR-21 overexpression had no effect on IP-10 mRNA levels (Figure 3B). Distinctively, the transfection of mimics greatly inhibited the IP-10 protein released from LPS-stimulated THP-1 cells $(P=0.041$; Figure 3C), indicated that miR-21 directly targeting IP-10 at the post-transcriptional level. IP-10 as an inflammatory cytokine is almost undetectable within the supernatant of THP-1 cells, and its secretion can be stimulated by LPS.

To further evaluate the relationship between miR-21 and IP-10, miR-21 inhibitors were transfected to THP-1 cells (Figure 3D). In line with the abovementioned trends, miR-21 inhibitors did not significantly increase the IP-10 mRNA expression but efficiently enhanced IP-10 production in THP-1 cells after LPS stimulation $(P=0.015$; Figures 3E,F). The miRNA inhibited the level of the
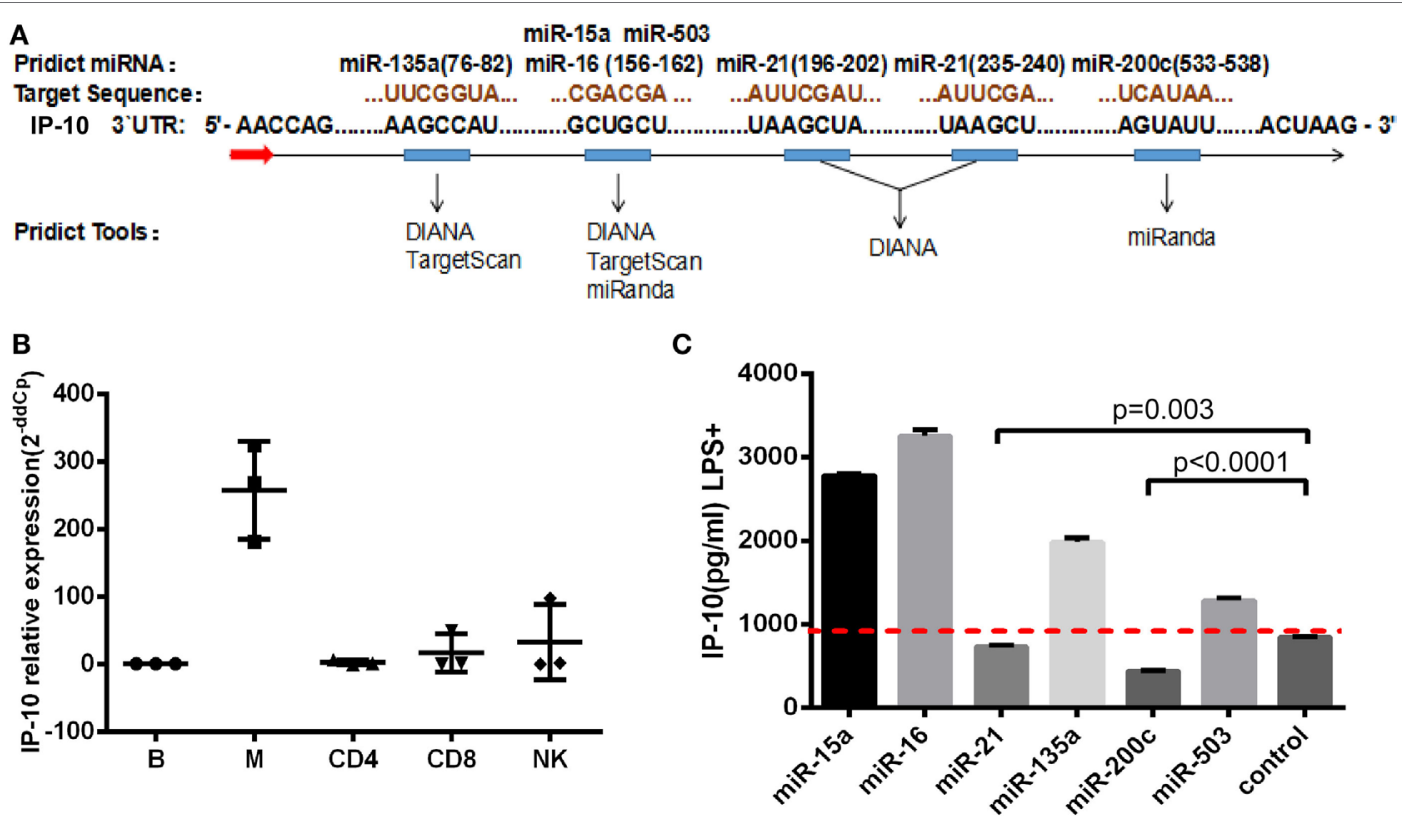

FIGURE 2 | miR-21 identified as potential regulator of interferon-inducible protein 10 (IP-10) secretion. miRNA candidates were screened, using three targetprediction software programs, including TargetScan, miRanda, and DIANA for binding sites. (A) miRNAs and IP-10 3'UTR matching sequences; (B) IP-10 mRNA expression exhibited by B cells, monocytes, CD4 ${ }^{+}$and CD8 ${ }^{+}$T cells, and NK cells; (C) THP-1 cells were transfected with either mimics of six miRNAs or negative control for $48 \mathrm{~h}$ and stimulated by lipopolysaccharide (LPS) for $24 \mathrm{~h}$. IP-10 production in supernatants of THP-1 cells was measured. 
A

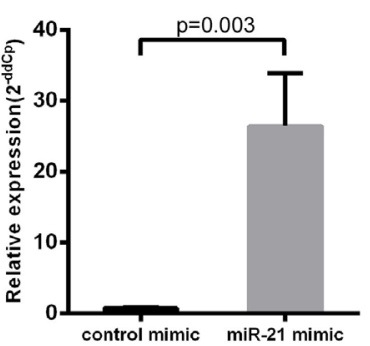

D

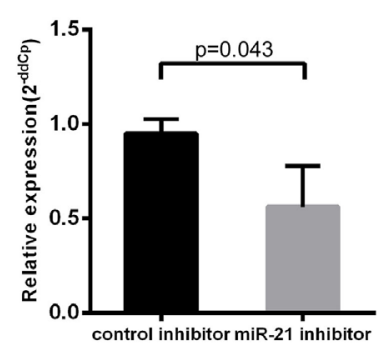

G

miR-21 3 ' - AgUUGUAGUCAGACUAUUCGAUC - 5 '

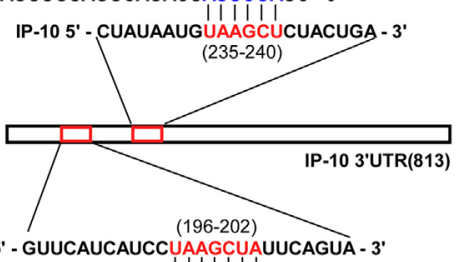

IP-10 5' - GUUCAUCAUCCUAAGCUAUUCAGUA -

miR-21 3' - AgUUGUAGUCAGACUAUUCGAUC - 5'
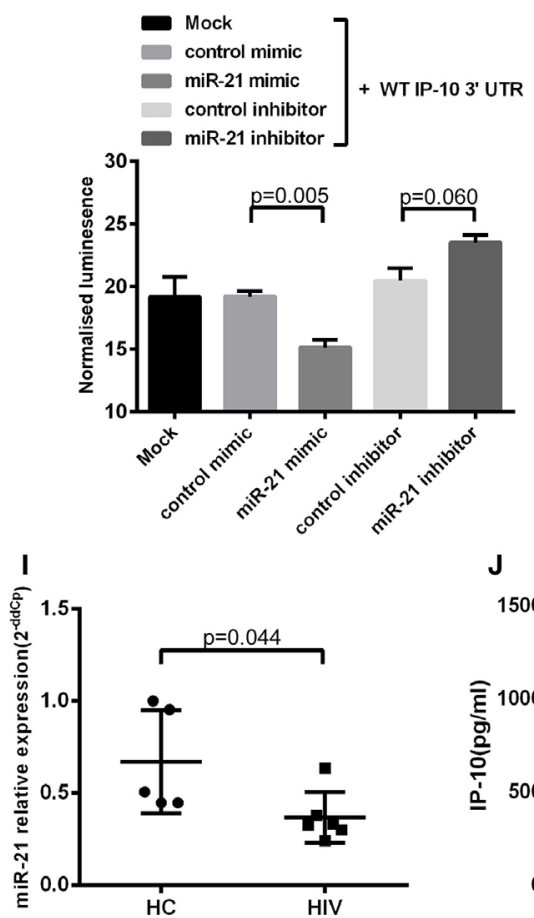

J

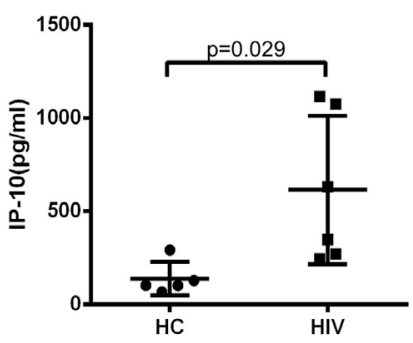

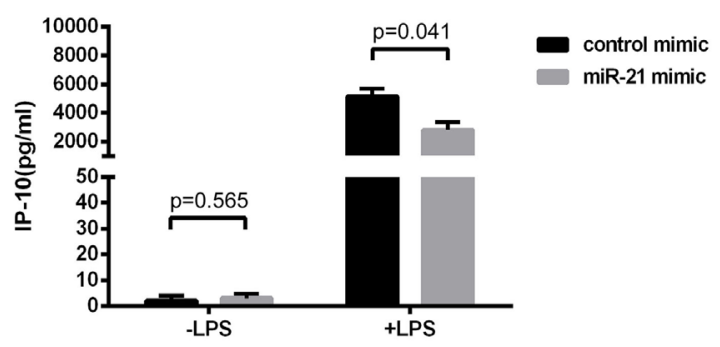

$\mathbf{F}$

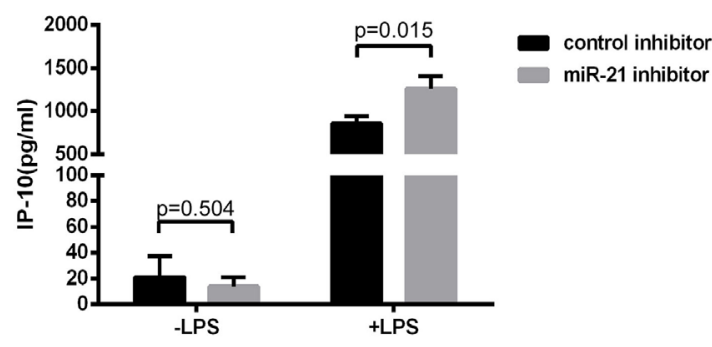

H

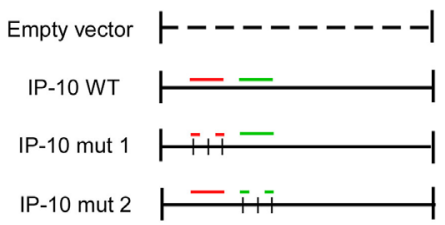

Empty vector

WT

Mut-1 + miR-21 mimic

Mut-2

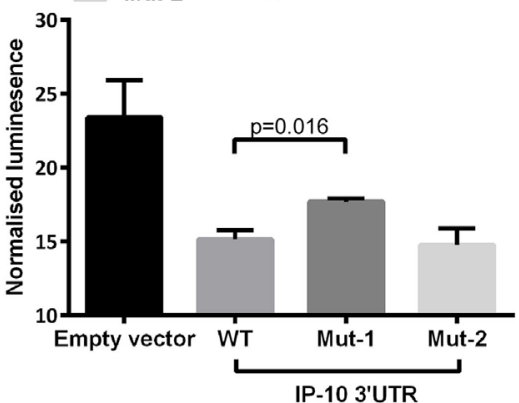

K

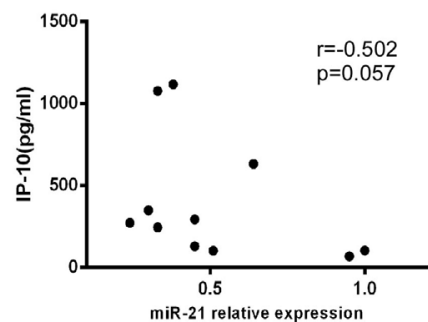


FIGURE 3 | Continued

miR-21 regulates interferon-inducible protein 10 (IP-10) secretion in THP-1 cells. THP-1 cells were transfected with either miR-21 mimics or control for $48 \mathrm{~h}$ and subsequently stimulated by lipopolysaccharide (LPS) for another $24 \mathrm{~h}$. (A) The transfection of miR-21 mimics led to enhanced expression of miR-21 in THP-1 cells. Following the transfection, IP-10 mRNA expression (B) and secretion after LPS stimulation (C) were measured. THP-1 cells were transfected with either miR-21 inhibitors or control over $48 \mathrm{~h}$ and stimulated by LPS for $24 \mathrm{~h}$. (D) The transfection of miR-21 inhibitors triggered a decreased expression of miR-21 in THP-1 cells. The inhibition of miR-21 did not influence IP-10 mRNA levels (E) yet did increase IP-10 secretion (F). (G) Two conserved miR-21 binding sites located at the IP-10 3'UTR. Luciferase activity was significantly lower in mimic-treated cells but was slightly higher in fluorescence intensity of inhibitor-treatment cells than in the mock cells. (H) Co-transfected with miR-21 mimic and IP-10 3'UTR mut-2 (235-240), not mut-1 (196-202) vector showed more downregulated luciferase expression than the empty vector control. (I) Comparison of miR-21 expression in sorted monocytes from HIV and healthy controls (HCs). (J) Comparison of plasma IP-10 levels in HIV and HC. (K) Correlation between the miR-21 expression in CD14+ monocytes and plasma IP-10 level.

target gene through two main mechanisms, including mRNA degradation and the blockade of translation at the steps of initiation or elongation (26). Our results suggest that miR-21 altered the protein, but not mRNA expression level of IP- 10 .

\section{Identification of IP-10 As Direct Target of miR-21}

We explored the potential of miR-21 to directly target the $3^{\prime} \mathrm{UTR}$ of IP-10. The two putative binding sites between IP-10 3'UTR and miR-21 are shown in Figure 3G. Luciferase report vectors carrying the full 3'UTR of wild-type (WT) IP-10, mutation site 1 (mut-1, 196-202), or mutation site 2 (mut-2, 235-240) were constructed. Reporters were transfected into $293 \mathrm{~T}$ cells with either miR-21 mimics or inhibitors, and luminescence in miR-21 mimic-treated cells was clearly less than in controls $(P=0.005)$. Furthermore, increasing fluorescent properties were ascribed to the suppression of endogenous miR-21, a phenomenon not observed in the controls $(P=0.060$; Figure 3G). The results show that miR-21 can directly target the $3^{\prime}$ UTR of IP-10 and affect subsequent transcription. Therefore, we also worked to identify the effective binding site between IP-10 and miR-21. No substantial differences in the inhibition luciferase activity between the WT reporter and mut-2 were observed, and transfection with mut-1 reporter had no impact on luciferase activity (Figure $3 \mathbf{H}$ ). According to our results, miR-21 can directly target IP-10 3'UTR through bind site 1 (196-202).

\section{miR-21 Is Downregulated in Monocyte of HIV-Infected Patients}

We have demonstrated the miR-21 potential to regulate IP-10 secretion. Further studies on the association of increased plasma levels of IP-10 observed in HIV-infected patients with miR-21 alteration in primary monocytes were performed. Because monocyte is a main source of IP-10 (16), we sorted monocytes from HIV-infected individuals $(n=6)$ as well as HCs $(n=5)$ to detect the relative expression of miR-21. The levels of miR-21 in monocytes of HIV-infected patients were considerably lower $(P=0.044$; Figure 3I) than those of HCs, whereas IP-10 levels in plasma exhibited the opposite tendency $(P=0.029$, Figure 3J). There was a trend of negative correlation between miR-21 expression in monocytes and plasma IP- 10 concentrations $(r=-0.502$, $P=0.057$; Figure 3K). We found that overexpression of miR-21 in primary monocytes $(P=0.002$; Figure S2A in Supplementary Material) from $\mathrm{HIV}$-infected patients has the decreasing tendency of IP-10 production by LPS stimulation ( $P=0.057$; Figure S2B in Supplementary Material). The results suggesting that miR-21 may contribute to the regulation of IP-10 production in monocytes in the context of HIV infection.

\section{Elevated Expression of ISG15 Weakens the Regulation of IP-10 by miR-21 in THP-1-MA Cells}

Monocytes circulating in the blood stream continuously repopulate the macrophage population that resides within tissues (27). The vast majority of transcription factors are altered in the process of the differentiation monocyte to macrophage (28), which is an important source of the release of IP-10 (29). Therefore, we detected the alterations of miR-21 and IP-10 during the differentiation process from THP-1 to THP-1-MA cells. As illustrated in Figures 4A,B, the expression of miR-21 and IP-10 was remarkably more enhanced in THP-1-MA than in THP-1 cells $(P=0.002$ and $P=0.037$, respectively). We subsequently measured the IP-10 levels of unstimulated and stimulated THP-1 and THP-1-MA cells. The results showed a similar trend of increase in IP-10 secretion within the supernatant of THP-1-MA cells over that in THP-1 cells $(P=0.0008)$, especially after LPS stimulation $(P=0.0004$; Figure $4 \mathrm{C})$. We here asked why the increase of miR-21 during monocyte-macrophage differentiation was not accompanied by IP-10 inhibition, and we also wished to determine the potential of miR-21 to suppress IP-10 secretion in macrophages. In the results, we observed that miR-21 mimics or inhibitors did not play a significant role in IP-10 secretion of LPS-stimulated THP-1-MA cells (Figures 4D,E).

We then explored the potential mechanisms controlling the differential effects of miR-21 on IP-10 production between monocytes and macrophages. We speculated that there may have been some alteration of particular factors along the IP-10 signaling pathway altered in the THP-1-MA cells compared with the THP-1 cells, which may have attenuated the regulatory effect of miR-21 on IP-10 secretion (29). We found that IFN-stimulated gene 15 (ISG15), an IP-10 upstream molecule, underwent expression changes during the process of differentiation from THP-1 to THP1-MA (30). ISG15 may trigger IP-10 expression by its function on NF- $\mathrm{B}$ (31-33), which is a crucial transcription factor for IP-10 induction (34-37). This prompted us to investigate the role played by the enhanced level of ISG15 on IP-10 expression within THP1 -MA by comparison with the THP-1 cells ( $P=0.010$; Figure 4 F). We propose that, through NF-kB activation, ISG15 regulates IP-10 at the transcription level. We found that the successful inhibition of ISG15 by siRNA ( $P=0.017$; Figure 4G) did significantly decrease both IP-10 mRNA expression $(P=0.039$; Figure $4 \mathbf{H})$ and protein production $(P=0.021$; Figure $4 \mathrm{I})$. This is different for miR-21. 


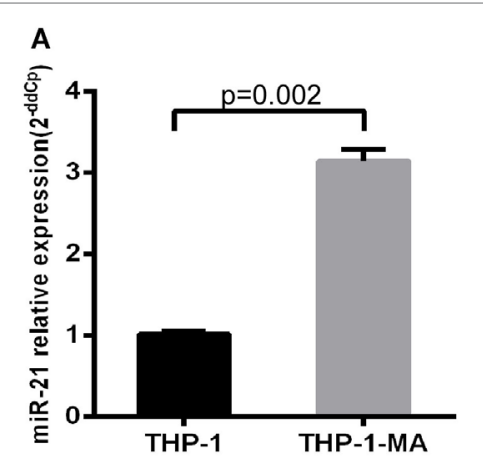

D

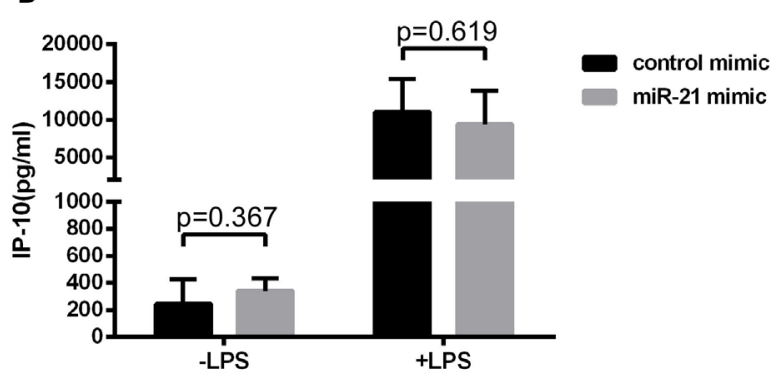

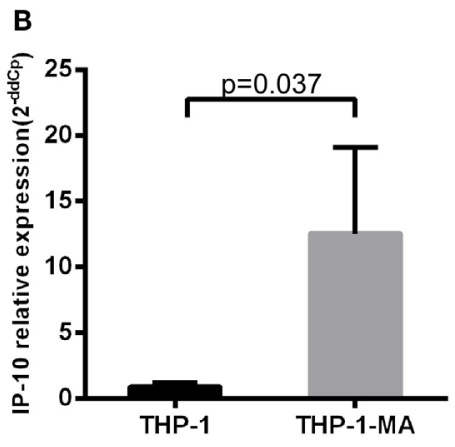

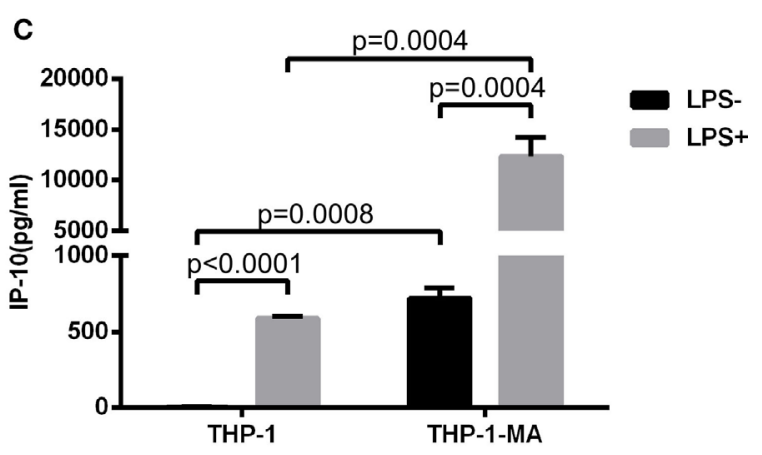

E
F

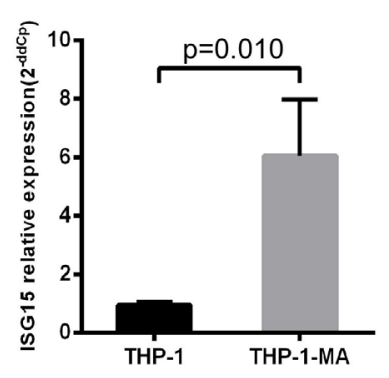

G

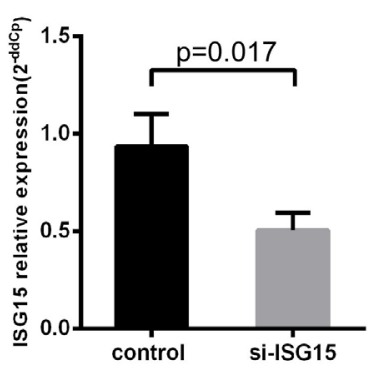

H

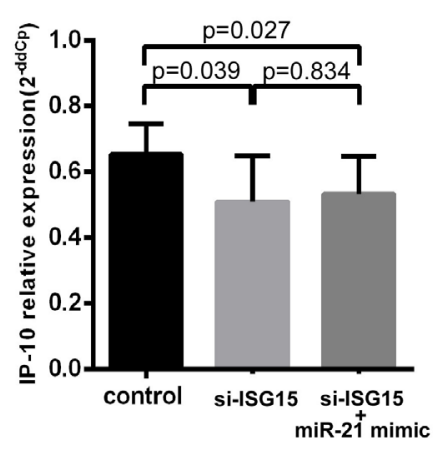

I

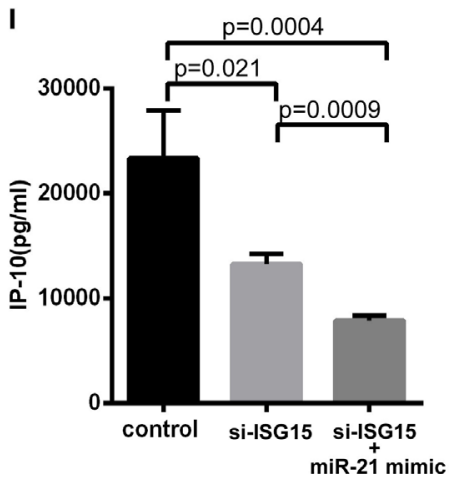

FIGURE 4 | IFN-stimulated gene 15 (ISG15) weakens the function of miR-21 regulation to IP-10. Comparison of miR-21 expression (A), IP-10 mRNA level (B), and IP-10 secretion (C) in THP-1 and THP-1 macrophages (THP-1-MA) cells. (D) Efficient overexpression of miR-21 did not reduce the secretion level of IP-10 in THP-1-MA cells stimulated by lipopolysaccharide (LPS). (E) Transfection with miR-21 inhibitors did not promote interferon-inducible protein 10 (IP-10) secretion in THP-1-MA cells stimulated by LPS. (F) Comparison of the relative expressions of ISG15 in THP-1 and THP-1-MA cells. (G) The suppression of ISG15 mRNA expression achieved by transfection of si-ISG15 to THP-1-MA cells. (H,I) Relative expression of IP-10 mRNA or IP-10 secretion after transfection of si-ISG15 itself, or with miR-21 mimics in the LPS-stimulated THP-1-MA cells.

Our data indicated that post-transcriptional regulation carried out by miR-21 only acts on IP-10 protein production, but not IP-10 mRNA (Figures 3A-F). We found that the effect of miR-21 on IP-10 protein production becomes prominent after ISG-15 was silenced $(P=0.0009$; Figure $4 \mathrm{I})$. The results indicated that the transcriptional activation induced by enhanced ISG15 production is stronger than fine-tuning effect of post-transcriptional regulation mediated by miR-21. Therefore, overexpression of miR-21 cannot reduce the IP-10 protein production efficiently in THP-1-MA cells where ISG15 is highly expressed. Comparing to THP-1-MA cells, THP-1 cells express less ISG15 (Figure 4F). Nevertheless, suppression of ISG15 in THP-1 cells still led to reduced IP-10 mRNA expression $(P=0.014)$ and IP-10 protein production $(P=0.010)$ (Figures S3A,B in Supplementary Material). It is interesting to note that, since the basal level of ISG15 is reduced in THP-1 cells, we also observed that the silencing of ISG15 has a smaller effect on IP-10 production in comparison to the relative change in THP-1-MA cells (Figure S3C in Supplementary Material). These findings support the thesis that enhanced ISG15 levels in THP-1-MA facilitate IP-10 production and, therefore, overcome the inhibition of IP-10 by miR-21, potentially accounting for the differential effect of miR-21 to IP-10 expression in monocytes and macrophages.

\section{DISCUSSION}

HIV-1-associated immune dysregulation and activation is presumably established during the early stage of HIV-1 infection 
when a host of plasma cytokines and chemokines are rapidly developed $(16,38,39)$. IP-10 is a pro-inflammatory chemokine expressed within a variety of cell types with pleiotropic function (40) and plays a pivotal role in regulating monocyte-mediated inflammatory responses as well as enhanced immune activation (41), thereby facilitating the disease progression of HIV $(6,10,12,13)$. In this study, we identified miR-21 as an important regulator of IP-10 in monocytes at post-transcriptional regulation level. For the THP-1-MA cells, this regulation is weakened as ISG15 is strengthened.

In this study, we first demonstrated that miR-21 regulates IP-10 secretion in monocytes. In our screening of six miRNA potential candidates, we observed that IP-10 in supernatant declined following the overexpression of miR-21 in LPSstimulated THP-1 cells, and vice versa. Luciferase assay revealed that miR-21 directly targets IP-10 3'UTR through a predicted binding site (196-202). Previous studies performed on porcine kidney 15 cell lines and peritoneal macrophages from Sprague Dawley rats have showed that IP-10 was targeted by miR-21 (42, 43). Monocytes are one of the main sources of IP-10 (16), no study examining the post-transcriptional regulation of IP-10 in human monocytes has been reported to our knowledge. Therefore, using the THP-1 cell line, we found and report herein for the first time that miR-21 can regulate the expression of IP-10 in human monocytes. In HIV infection, it has been reported that increased circulating miR-21 in sera was able to discriminate HIV-infected from the HIV-uninfected groups (44) and correlated with soluble biomarkers of immune activation, including D-dimer and IL-6 (45). But whether miR-21 was altered in primary monocyte in HIV infection has not been reported. Our subsequent analyses of primary monocytes from HIV-infected patients showed the decreased expression of miR-21 and a trend of variation in miR-21 expression opposite to IP-10 levels in plasma. And transfection of miR-21 mimics has the tendency to decrease the LPS-stimulated IP-10 production by primary monocytes from HIV-infected patients. The result suggests that miR-21 may have the potential to regulate IP-10 production by monocyte in HIV infection. IP-10 promotes inflammation and activation, which associate with HIV disease progression and are difficult to be completely eliminated in HIV infection $(2-4,6,10-12)$. We found that miR-21 could effectively inhibit the expression of IP-10 by monocytes, which is an important source of inflammation in HIV infection (46). Providing that the miRNA oligonucleotides (e.g., miR-122) have been previously employed in clinical control of infectious diseases (47), our results indicate the potential role of miR-21 as complementary therapeutic regimen for HIV infection by controlling the level of inflammation.

Although we have observed the importance of the role of miR-21 in regulating IP-10 at the post-transcriptional level of monocytes, this relationship was not observed in THP-1-MA cells. We detected the levels of expression of miR-21 and IP-10 during the differentiation process from monocyte to macrophage. We found both miR-21 and IP-10 were upregulated during the differentiation from THP-1 to THP-1-MA. This change in IP-10 expression may benefit the secretion of IP-10 and promote proinflammatory activation. However, it has not yet been satisfactorily explained why the effective overexpression of miR-21 did not reduce the generation of IP-10 in THP-1-MA cells in our study. We postulated that during the differentiation of monocyte to macrophage, some key genes regulating the expression of IP-10 altered and this weakens the regulation of miR-21 to IP-10. By exploring the signaling pathway and reviewing previous reports, we proposed an ubiquitin-like protein, ISG15 as essential in regulating the IP-10 expression and it exhibited higher levels during monocyte-macrophage differentiation and HIV infection $(30,48)$. Recent study revealed that ISG15 can induce the expression of IP-10 in Candida albicans infection (49). NF- $\mathrm{BB}$ is a well-known transcription factor for IP-10 gene activation (34-37). Previous studies showed that ISG15 enhances NF- $\mathrm{B}$ activation by negatively regulates protein phosphatase $2 \mathrm{C} \beta(\mathrm{PP} 2 \mathrm{C} \beta)$ activity (31), which downregulated NF- $\mathrm{KB}$ activation by altering IKK activity (33). We postulated ISG15 may increase IP-10 production by the activation of NF- $\kappa$ B in THP-1-MA cells. Simmons et al. have described a connection between the plasma levels of IP-10 and ISG15 expression in HIV infection (16). According to our results, the process of cell differentiation from THP-1 to THP-1-MA is characterized by a conspicuous increase of ISG15 expression. The inhibition of ISG15 expression by siRNA triggers the recovery of miR-21 function to suppress IP-10 secretion in THP-1-MA cells. We proposed here that IP-10 is under the control of both ISG15 and miR-21. miR-21 directly targeting IP-10 at the post-transcriptional level while IP-10 is also regulated by ISG15 independently at the transcription level. The impact of ISG15 and miR-21 on IP-10 protein production is not equal. Our data indicated that enhanced ISG15-mediated transcriptional activation can overcome the post-transcriptional suppression of miR-21 in THP-1-MA cells. When ISG-15 was silenced, the effect of miR-21 on IP-10 production becomes prominent. The current study offers a comprehensive understanding of the regulation of IP-10 in HIV infection.

To conclude, we report the potential of $\mathrm{miR}-21$ for regulating IP-10 expression in monocytes, whereas the elevated expression of ISG15 weakens the regulation of IP-10 by miR-21 in macrophages. Upregulated IP-10 in HIV infections is associated with high inflammation levels, rapid loss of $\mathrm{CD} 4^{+} \mathrm{T}$ cells, promoting infection by enabling viral reservoirs. Therefore, reducing IP-10 production is a promising and critical way of controlling HIVassociated inflammation and disease progression. Our results deepen the understanding of HIV pathogenesis and pave the way for inflammation intervention in HIV infection.

\section{ETHICS STATEMENT}

The ethics approval was obtained from the First Hospital of China Medical University, and all the investigated participants were informed about the collection of blood samples, and provided written consent prior to enrolment in the study.

\section{AUTHOR CONTRIBUTIONS}

HS, Z-NZ, and XW conceived and designed the experiments; $\mathrm{XW}$ and L-LZ performed the experiments; XW analyzed the 
data; L-BY, Y-JF, Y-JJ, H-BD, and Z-XC contributed reagents/ materials/analysis tools; XW and Z-NZ wrote the paper.

\section{FUNDING}

This study was supported by grants from the National Natural Science Foundation of China (81371884), the Mega-Projects of National Science Research for the 13th Five-Year Plan

\section{REFERENCES}

1. Rajasuriar R, Khoury G, Kamarulzaman A, French MA, Cameron PU, Lewin SR. Persistent immune activation in chronic HIV infection: do any interventions work? AIDS (2013) 27(8):1199-208. doi:10.1097/ QAD.0b013e32835ecb8b

2. Deeks SG, Tracy R, Douek DC. Systemic effects of inflammation on health during chronic HIV infection. Immunity (2013) 39(4):633-45. doi:10.1016/j. immuni.2013.10.001

3. Hileman CO, Funderburg NT. Inflammation, immune activation, and antiretroviral therapy in HIV. Curr HIV/AIDS Rep (2017) 14(3):93-100. doi:10.1007/ s11904-017-0356-x

4. Lederman MM, Funderburg NT, Sekaly RP, Klatt NR, Hunt PW. Residual immune dysregulation syndrome in treated HIV infection. Adv Immunol (2013) 119:51-83. doi:10.1016/B978-0-12-407707-2.00002-3

5. Psomas C, Younas M, Reynes C, Cezar R, Portales P, Tuaillon E, et al. One of the immune activation profiles observed in HIV-1-infected adults with suppressed viremia is linked to metabolic syndrome: the ACTIVIH study. EBioMedicine (2016) 8:265-76. doi:10.1016/j.ebiom.2016.05.008

6. Jiao Y, Zhang T, Wang R, Zhang H, Huang X, Yin J, et al. Plasma IP-10 is associated with rapid disease progression in early HIV-1 infection. Viral Immunol (2012) 25(4):333-7. doi:10.1089/vim.2012.0011

7. Norris PJ, Pappalardo BL, Custer B, Spotts G, Hecht FM, Busch MP. Elevations in IL-10, TNF-alpha, and IFN-gamma from the earliest point of HIV Type 1 infection. AIDS Res Hum Retroviruses (2006) 22(8):757-62. doi:10.1089/aid.2006.22.757

8. Stylianou E, Bjerkeli V, Yndestad A, Heggelund L, Waehre T, Damas JK, et al. Raised serum levels of interleukin-18 is associated with disease progression and may contribute to virological treatment failure in HIV-1infected patients. Clin Exp Immunol (2003) 132(3):462-6. doi:10.1046/j. 1365-2249.2003.02179.x

9. Ahmadi Z, Arababadi MK, Hassanshahi G. CXCL10 activities, biological structure, and source along with its significant role played in pathophysiology of type I diabetes mellitus. Inflammation (2013) 36(2):364-71. doi:10.1007/ s10753-012-9555-1

10. Liovat AS, Rey-Cuille MA, Lecuroux C, Jacquelin B, Girault I, Petitjean G, et al. Acute plasma biomarkers of $\mathrm{T}$ cell activation set-point levels and of disease progression in HIV-1 infection. PLoS One (2012) 7(10):e46143. doi:10.1371/journal.pone.0046143

11. Ploquin MJ, Madec Y, Casrouge A, Huot N, Passaes C, Lecuroux C, et al. Elevated basal pre-infection CXCL10 in plasma and in the small intestine after infection are associated with more rapid HIV/SIV disease onset. PLoS Pathog (2016) 12(8):e1005774. doi:10.1371/journal.ppat.1005774

12. Noel N, Boufassa F, Lecuroux C, Saez-Cirion A, Bourgeois C, Dunyach-Remy C, et al. Elevated IP10 levels are associated with immune activation and low CD4(+) T-cell counts in HIV controller patients. AIDS (2014) 28(4):467-76. doi:10.1097/QAD.0000000000000174

13. Ramirez LA, Arango TA, Thompson E, Naji M, Tebas P, Boyer JD. High IP-10 levels decrease T cell function in HIV-1-infected individuals on ART. J Leukoc Biol (2014) 96(6):1055-63. doi:10.1189/jlb.3A0414-232RR

14. Wang Z, Wu T, Ma M, Zhang Z, Fu Y, Liu J, et al. Elevated interferon-gamma-induced protein 10 and its receptor CXCR3 impair NK cell function during HIV infection. JLeukoc Biol (2017) 102(1):163-70. doi:10.1189/ jlb.5A1016-444R

15. Lane BR, King SR, Bock PJ, Strieter RM, Coffey MJ, Markovitz DM. The C-X-C chemokine IP-10 stimulates HIV-1 replication. Virology (2003) 307(1):122-34. doi:10.1016/S0042-6822(02)00045-4
(2017ZX10201101), and the Mega-Projects of National Science Research for the 12th Five-Year Plan (2012ZX10001-006).

\section{SUPPLEMENTARY MATERIAL}

The Supplementary Material for this article can be found online at http://journal.frontiersin.org/article/10.3389/fimmu.2017.01122/ full\#supplementary-material.

16. Simmons RP, Scully EP, Groden EE, Arnold KB, Chang JJ, Lane K, et al. HIV-1 infection induces strong production of IP-10 through TLR7/9-dependent pathways. AIDS (2013) 27(16):2505-17. doi:10.1097/01.aids.0000432455.06476.bc

17. Pujantell M, Badia R, Ramirez C, Puig T, Clotet B, Ballana E, et al. Long-term $\mathrm{HIV}-1$ infection induces an antiviral state in primary macrophages. Antiviral Res (2016) 133:145-55. doi:10.1016/j.antiviral.2016.08.004

18. Swaminathan S, Murray DD, Kelleher AD. The role of microRNAs in HIV-1 pathogenesis and therapy. AIDS (2012) 26(11):1325-34. doi:10.1097/ QAD.0b013e328352adca

19. Swaminathan S, Murray DD, Kelleher AD. miRNAs and HIV: unforeseen determinants of host-pathogen interaction. Immunol Rev (2013) 254(1):265-80. doi:10.1111/imr.12077

20. Swaminathan S, Kelleher AD. MicroRNA modulation of key targets associated with T cell exhaustion in HIV-1 infection. Curr Opin HIV AIDS (2014) 9(5):464-71. doi:10.1097/COH.0000000000000089

21. Amado T, Schmolka N, Metwally H, Silva-Santos B, Gomes AQ. Crossregulation between cytokine and microRNA pathways in $\mathrm{T}$ cells. Eur J Immunol (2015) 45(6):1584-95. doi:10.1002/eji.201545487

22. Swaminathan S, Suzuki K, Seddiki N, Kaplan W, Cowley MJ, Hood CL, et al. Differential regulation of the Let-7 family of microRNAs in CD4+ T Cells Alters IL-10 expression. J Immunol (2012) 188(12):6238-46. doi:10.4049/ jimmunol.1101196

23. Zhang Y, Yin Y, Zhang S, Luo H, Zhang H. HIV-1 infection-induced suppression of the Let-7i/IL-2 axis contributes to CD4(+) T cell death. Sci Rep (2016) 6:25341. doi:10.1038/srep25341

24. Tsuchiya S, Kobayashi Y, Goto Y, Okumura H, Nakae S, Konno T, et al. Induction of maturation in cultured human monocytic leukemia cells by a phorbol diester. Cancer Res (1982) 42(4):1530-6.

25. Tsai JH, Kuo CH, Yang P, Cheng KH, Wang PW, Chen CC, et al. Effects of antidepressants on IP-10 production in LPS-activated THP-1 human monocytes. Int J Mol Sci (2014) 15(8):13223-35. doi:10.3390/ijms150813223

26. Inui M, Martello G, Piccolo S. MicroRNA control of signal transduction. Nat Rev Mol Cell Biol (2010) 11(4):252-63. doi:10.1038/nrm2868

27. Davies LC, Taylor PR. Tissue-resident macrophages: then and now. Immunology (2015) 144(4):541-8. doi:10.1111/imm.12451

28. Liu H, Shi B, Huang CC, Eksarko P, Pope RM. Transcriptional diversity during monocyte to macrophage differentiation. Immunol Lett (2008) 117(1):70-80. doi:10.1016/j.imlet.2007.12.012

29. Tighe RM, Liang J, Liu N, Jung Y, Jiang D, Gunn MD, et al. Recruited exudative macrophages selectively produce CXCL10 after noninfectious lung injury. Am J Respir Cell Mol Biol (2011) 45(4):781-8. doi:10.1165/rcmb.2010-0471OC

30. Goujon C, Moncorge O, Bauby H, Doyle T, Ward CC, Schaller T, et al. Human MX2 is an interferon-induced post-entry inhibitor of HIV-1 infection. Nature (2013) 502(7472):559-62. doi:10.1038/nature12542

31. Takeuchi T, Kobayashi T, Tamura S, Yokosawa H. Negative regulation of protein phosphatase 2Cbeta by ISG15 conjugation. FEBS Lett (2006) 580(18):4521-6. doi:10.1016/j.febslet.2006.07.032

32. Darb-Esfahani S, Sinn BV, Rudl M, Sehouli J, Braicu I, Dietel M, et al. Interferon-stimulated gene, $15 \mathrm{kDa}$ (ISG15) in ovarian high-grade serous carcinoma: prognostic impact and link to NF-kappaB pathway. Int J Gynecol Pathol (2014) 33(1):16-22. doi:10.1097/PGP.0b013e31827b25a2

33. Prajapati S, Verma U, Yamamoto Y, Kwak YT, Gaynor RB. Protein phosphatase 2Cbeta association with the IkappaB kinase complex is involved in regulating NF-kappaB activity. J Biol Chem (2004) 279(3):1739-46. doi:10.1074/jbc. M306273200

34. Krinninger P, Brunner C, Ruiz PA, Schneider E, Marx N, Foryst-Ludwig A, et al. Role of the adipocyte-specific NF-kappaB activity in the regulation of IP-10 
and T cell migration. Am J Physiol Endocrinol Metab (2011) 300(2):E304-11. doi:10.1152/ajpendo.00143.2010

35. Zhou Y, Wang S, Ma JW, Lei Z, Zhu HF, Lei P, et al. Hepatitis B virus protein $\mathrm{X}$-induced expression of the CXC chemokine IP-10 is mediated through activation of NF-kappaB and increases migration of leukocytes. J Biol Chem (2010) 285(16):12159-68. doi:10.1074/jbc.M109.067629

36. Brownell J, Bruckner J, Wagoner J, Thomas E, Loo YM, Gale M Jr, et al. Direct, interferon-independent activation of the CXCL10 promoter by NF-kappaB and interferon regulatory factor 3 during hepatitis $\mathrm{C}$ virus infection. J Virol (2014) 88(3):1582-90. doi:10.1128/JVI.02007-13

37. Borgland SL, Bowen GP, Wong NC, Libermann TA, Muruve DA. Adenovirus vector-induced expression of the C-X-C chemokine IP-10 is mediated through capsid-dependent activation of NF-kappaB. J Virol (2000) 74(9):3941-7. doi:10.1128/JVI.74.9.3941-3947.2000

38. Stacey AR, Norris PJ, Qin L, Haygreen EA, Taylor E, Heitman J, et al. Induction of a striking systemic cytokine cascade prior to peak viremia in acute human immunodeficiency virus type 1 infection, in contrast to more modest and delayed responses in acute hepatitis B and C virus infections. J Virol (2009) 83(8):3719-33. doi:10.1128/JVI.01844-08

39. McMichael AJ, Borrow P, Tomaras GD, Goonetilleke N, Haynes BF. The immune response during acute HIV-1 infection: clues for vaccine development. Nat Rev Immunol (2010) 10(1):11-23. doi:10.1038/nri2674

40. Antonelli A, Ferrari SM, Giuggioli D, Ferrannini E, Ferri C, Fallahi P. Chemokine (C-X-C motif) ligand (CXCL)10 in autoimmune diseases. Autoimmun Rev (2014) 13(3):272-80. doi:10.1016/j.autrev.2013.10.010

41. Liu M, Guo S, Hibbert JM, Jain V, Singh N, Wilson NO, et al. CXCL10/IP-10 in infectious diseases pathogenesis and potential therapeutic implications. Cytokine Growth Factor Rev (2011) 22(3):121-30. doi:10.1016/j.cytogfr.2011.06.001

42. Yan $\mathrm{W}$, Chen $\mathrm{C}$, Chen H. Estrogen downregulates miR-21 expression and induces inflammatory infiltration of macrophages in polymyositis: role of CXCL10. Mol Neurobiol (2017) 54(3):1631-41. doi:10.1007/s12035-016-9769-6

43. Huang J, Ma G, Fu L, Jia H, Zhu M, Li X, et al. Pseudorabies viral replication is inhibited by a novel target of miR-21. Virology (2014) 45(6-457):319-28. doi:10.1016/j.virol.2014.03.032
44. Thapa DR, Hussain SK, Tran WC, D'Souza G, Bream JH, Achenback CJ, et al. Serum microRNAs in HIV-infected individuals as pre-diagnosis biomarkers for AIDS-NHL. J Acquir Immune Defic Syndr (2014) 66(2):229-37. doi:10.1097/QAI.0000000000000146

45. Murray DD, Suzuki K, Law M, Trebicka J, Neuhaus J, Wentworth D, et al. Circulating microRNAs in sera correlate with soluble biomarkers of immune activation but do not predict mortality in ART treated individuals with HIV-1 infection: a case control study. PLoS One (2015) 10(10):e0139981. doi:10.1371/ journal.pone. 0139981

46. Anzinger JJ, Butterfield TR, Angelovich TA, Crowe SM, Palmer CS. Monocytes as regulators of inflammation and HIV-related comorbidities during cART J Immunol Res (2014) 2014:569819. doi:10.1155/2014/569819

47. Janssen HL, Reesink HW, Lawitz EJ, Zeuzem S, Rodriguez-Torres M, Patel K, et al. Treatment of HCV infection by targeting microRNA. N Engl J Med (2013) 368(18):1685-94. doi:10.1056/NEJMoa1209026

48. Scagnolari C, Monteleone K, Selvaggi C, Pierangeli A, D'Ettorre G, Mezzaroma I, et al. ISG15 expression correlates with HIV-1 viral load and with factors regulating $\mathrm{T}$ cell response. Immunobiology (2016) 221(2):282-90. doi:10.1016/j.imbio.2015.10.007

49. Dong C, Gao N, Ross BX, Yu FX. ISG15 in host defense against Candida albicans infection in a mouse model of fungal keratitis. Invest Ophthalmol Vis Sci (2017) 58(7):2948-58. doi:10.1167/iovs.17-21476

Conflict of Interest Statement: The authors declare that the research was conducted in the absence of any commercial or financial relationships that could be construed as a potential conflict of interest.

Copyright $\odot 2017$ Wu, Zhang, Yin, Fu, Jiang, Ding, Chu, Shang and Zhang. This is an open-access article distributed under the terms of the Creative Commons Attribution License (CC BY). The use, distribution or reproduction in other forums is permitted, provided the original author(s) or licensor are credited and that the original publication in this journal is cited, in accordance with accepted academic practice. No use, distribution or reproduction is permitted which does not comply with these terms. 